\title{
Stable Electron Beams With Low Absolute Energy Spread From a Laser Wakefield Accelerator With Plasma Density Ramp Controlled Injection *
}

\author{
C.G.R. Geddes ${ }^{\dagger}$, E. Cormier-Michel ${ }^{\ddagger}$, E.Esarey ${ }^{\S}$, W.P. Leemans ${ }^{\Uparrow}$, K. Nakamura ${ }^{\|}$, D. Panasenko, \\ G.R. Plateau ** C.B. Schroeder, Cs. Toth, Lawrence Berkeley National Lab, Berkeley CA 94720 \\ J.R. Cary ${ }^{\dagger \dagger}$, Tech-X Corporation, Boulder CO 80303
}

\begin{abstract}
Laser wakefield accelerators produce accelerating gradients up to hundreds of $\mathrm{GeV} / \mathrm{m}$, and recently demonstrated $1-10 \mathrm{MeV}$ energy spread at energies up to $1 \mathrm{GeV}$ using electrons self-trapped from the plasma. Controlled injection and staging may further improve beam quality by circumventing tradeoffs between energy, stability, and energy spread/emittance. We present experiments demonstrating production of a stable electron beam near $1 \mathrm{MeV}$ with hundred-keV level energy spread and central energy stability by using the plasma density profile to control selfinjection, and supporting simulations. Simulations indicate that such beams can be post accelerated to high energies, potentially reducing momentum spread in laser accelerators by 100 -fold or more.
\end{abstract}

\section{INTRODUCTION}

Laser wakefield accelerators (LFWA) [1, 2] obtain high gradients using the electric field of a plasma wave (the wakefield) driven by an intense laser. In most present experiments $[3,4,5,6,7]$, electrons are trapped and accelerated by increasing plasma density to decrease the wake phase velocity ( $\sim$ the laser group velocity $v_{g}$ in uniform plasmas), or by increasing laser power, until the trapping threshold is reached. Because dephasing limits electron energy gain [2], low densities $\left(<10^{18} \mathrm{~cm}^{-3}\right)$ will be required to reach momenta beyond $1 \mathrm{GeV} / \mathrm{c}$, requiring very high laser powers to reach the trapping threshold.

Injection control independent of the density of the accelerating channel is considered to improve stability while increasing final energy. Such techniques are also needed to reduce the 1- $20 \mathrm{MeV} / \mathrm{c}$ momentum spread of present LWFA experiments (including initial colliding pulse $[8,9$, 10] controlled injection experiments [11]) and their 0.1-1 $\mathrm{MeV} / \mathrm{c}$ transverse momentum. The plasma wake wavelength, $\lambda_{p} \sim 10-100 \mu \mathrm{m}$ [2], then requires injection of a femtosecond electron bunch with femtosecond timing.

\footnotetext{
* Work supported by the U.S. Dept. of Energy contracts DE-AC0205CH11231, DE-FG03-95ER40926, DE-FG02-01ER41178, DE-FG0203ER83857, DOE SciDAC, INCITE, and NERSC programs, and NSF grant 0113907 and 0614001 .

† cgrgeddes@lbl.gov

$\ddagger$ also University of Nevada Reno

$\S$ also University of Nevada Reno

I also U.C. Berkeley, University of Nevada Reno

$\|$ also University Tokyo

**also École Polytechnique, France

${ }^{\dagger} \dagger^{\dagger}$ also U. Colorado Boulder
}

Theoretical studies indicate that plasma density gradients can control injection [12, 13, 14]: in a decreasing plasma density ramp (or downramp), $\lambda_{p}$ increases causing wake fronts to fall further behind the laser, reducing wake velocity and trapping threshold and offering control over trapping by tailoring the gradient. Here we report that use of a plasma density downramp to control trapping produced bunches at momenta near $1 \mathrm{MeV} / \mathrm{c}$, with $170 \mathrm{keV} / \mathrm{c}$ FWHM momentum spread, $20 \mathrm{keV} / \mathrm{c}$ central momentum stability and transverse momentum, and $2 \mathrm{mrad}(2 \mathrm{keV} / \mathrm{c})$ RMS pointing stability over hundreds of shots [15]. The beams have order $\mathrm{nC}$ charge, and $\mathrm{THz}$ measurements and simulations indicate that they are ultrafast, suitable as an injector for LWFAs with the potential to reduce energy spread of the accelerated beams from the percent level of current experiments to order of $100 \mathrm{keV}$ at $\mathrm{GeV}$ energies and beyond.

\section{RESULTS}

In the present experiments, the density profile of a thin slit gas jet oriented transversely to the laser pulse provided the density gradient. Focusing on the downstream edge produced a downramp in the laser propagation direction; focusing at the upstream edge produced an increasing or flat top density typical of previous experiments [3, 4, 6, 5]. A pulse from the multi-arm LOASIS [16] Ti:Sapphire chirped pulse amplification laser ionized the hydrogen gas and drove the plasma wake (Fig. 1). Peak laser power was $10 \mathrm{TW}$, pulse length $47 \mathrm{fs}$ FWHM, and the focused spot size of $7.5 \mu \mathrm{m}$ FWHM yielded intensity of $1.6 \times 10^{19} \mathrm{~W} / \mathrm{cm}^{2}$. Plasma density was measured on target shots by a frequency doubled transverse interferometer [17]. Peak plasma density was $2.2 \pm 0.3 \times 10^{19} \mathrm{~cm}^{-3}$, and the profile in the laser propagation direction was approximately Gaussian above the measurement threshold $\sim 2 \times 10^{18} \mathrm{~cm}^{-3}$, with a FWHM width of $750 \mu \mathrm{m} \pm 100 \mu \mathrm{m}$.

An integrating current transformer (ICT) monitored bunch charge, and a magnetic spectrometer with bend angle of $55^{\circ}$ was used to obtain the momentum spectrum. The spectrometer used a phosphor detector covering a range $\pm 14 \%$ about a central momentum determined by magnet current, and had resolution of $\pm 5 \%$. Gamma ray detectors monitored Bremsstrahlung radiation from electrons stopping in the beam dump, and the ratio of gamma signal to charge was used in tuning the accelerator to identify high energy electron production [18]. A $10 \mu \mathrm{m}$ silver coated nitrocellulose pelicle transmitted the electron bunch and di- 


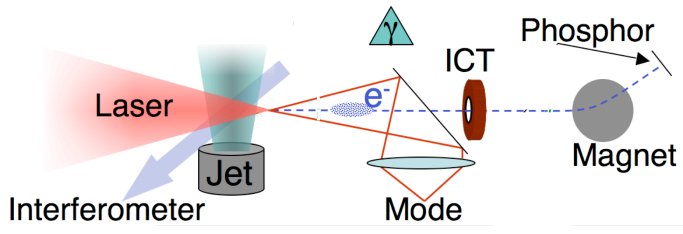

Figure 1: Setup for plasma ramp controlled electron injection. A laser is focused at the downstream edge of a thin slit gas jet oriented perpendicular to the pulse. Electrons are characterized by a magnetic spectrometer, ICT, and gamma detectors. The plasma profile is measured by a transverse interferometer. Transmitted laser light is diverted by a pellicle and imaged by a mode imager CCD.

verted the laser to a mode imager CCD [17] which imaged the transmitted laser spot profile at the plasma exit.

Scanning gas jet position along the laser propagation axis (Fig. 2A) controlled trapping. Focusing the laser on the upstream edge of the jet produced high momentum electrons via conventional self-modulated/self-trapped acceleration $[18,19]$, evidenced by high gamma signals. The magnetic spectrum was typical of unguided self-modulated experiments $[18,3,19]$, with an exponentially decaying spectrum reaching tens of $\mathrm{MeV}$. The mode imager showed strong self-modulation and filamentation of the laser mode into multiple spots, consistent with past experiments [19]. In contrast, focusing the laser near the downstream edge slightly increased bunch charge, but gamma signal dropped more than $70 \%$, indicating lower momentum electrons. Charge measured by the ICT was more than $3 \mathrm{nC}$ and charge stability improved, displaying fluctuations of only $\pm 15 \%$ RMS. Mode imager data showed that transmitted mode structure was similar to the vacuum mode, with no filamentation. Data on a subsequent run demonstrated more than $70 \%$ of the laser intensity was transmitted when using a prepulse to ionize the gas jet [17].

The magnetic spectrum showed that focusing at the downstream edge produced stable electron bunches with an order of magnitude lower absolute momentum spread than previously observed in laser accelerators (Fig. 2B). The central momentum is stable at $0.76 \mathrm{MeV} / \mathrm{c} \pm 20 \mathrm{keV} / \mathrm{c}$ RMS, and momentum spread is also stable at $170 \mathrm{keV} / \mathrm{c}$ FWHM $\pm 20 \mathrm{keV}$ RMS over 28 sequential (45 total) diagnostic shots. Charge fluctuated $40 \%$ RMS, though charge stability at the $15-25 \%$ level was observed earlier in the day; this variation in bunch stability is likely due to diurnal variation in laser stability. In the undispersed plane of the magnetic spectrometer, vertical bunch divergence was measured to be $20 \mathrm{mrad}$ FWHM $(\sim 20 \mathrm{keV} / \mathrm{c})$ with $1.8 \mathrm{mrad}$ RMS deviation, and pointing displayed $1.5 \mathrm{mrad}$ RMS stability. Removing the mode imager pellicle reduced the FWHM divergence in the undispersed direction by $10-20 \%$, but did not affect momentum spread. Combining data from three runs spanning 7 days of clock time, central momentum was stable between 0.76 and 0.78 $\mathrm{MeV} / \mathrm{c}$, FWHM energy spread was between 160 and 190
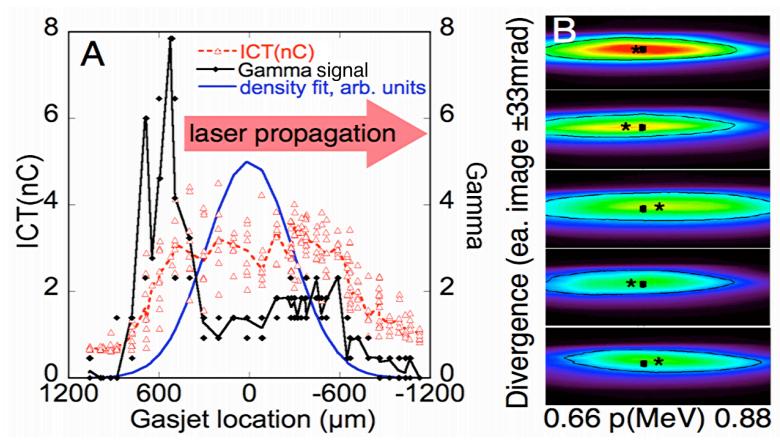

Figure 2: Stable beams produced by down ramp trapping. Scanning the jet position with respect to the laser focus (A) gives conventional self-modulated acceleration with the laser focus near the upstream (+) edge (high gamma signal, electrons to tens of $\mathrm{MeV}$ ), and stable low energy bunches from down ramp trapping at the down stream edge ( $\sim-300 \mu \mathrm{m}$, low gamma signal). A Gaussian fit to the plasma density is displayed on the same scale (A.U.). Several single shot magnetic spectra from sequential shots at jet position $-300 \mu \mathrm{m}$ (B) show stable beams near 0.76 $\mathrm{MeV} / \mathrm{c}$ with $\pm 10 \%$ momentum spread and $\pm 3 \%$ central momentum stability. The centroid $(*)$ is indicated with respect to the average centroid over 48 shots (square). The contour scale is fixed, showing the charge fluctuation ( $40 \%$ in this case; as low as $15 \%$ was observed).

$\mathrm{keV} / \mathrm{c}$, and divergence was between 17 and $23 \mathrm{mrad}$. The momentum spread, and the transverse momentum inferred from the divergence, are both 10 to 100 -fold below the values obtained in homogeneous plasmas. Correlation of phosphor and ICT measurements indicated charge in the $\mathrm{MeV}$ beam is $0.3-1 \mathrm{nC}$.

Downramp trapping was modeled using 2-D particle in cell simulations (VORPAL code [20]) with parameters similar to the experiments (related: [12, 13, 14]). Use of a large physical domain of $200 \mu \mathrm{m}(240 \mu \mathrm{m})$ with 6000 (900) cells in the longitudinal (transverse) direction and 5 particles per cell was required. The plasma shape was Gaussian, with peak density of $1.8 \times 10^{19} \mathrm{~cm}^{-3}$, and a FWHM of $940 \mu \mathrm{m}$. The simulation started $750 \mu \mathrm{m}$ into the ramp to reduce simulation size. The laser was focused $600 \mu \mathrm{m}$ downstream of the center of the Gaussian, within the range that produced $\mathrm{MeV}$ bunches experimentally (Fig. 2).

The simulations show trapping of an electron bunch near the end of the jet, where density is $\sim 0.5-1 \times 10^{18} \mathrm{~cm}^{-3}$ such that the plasma wavelength is resonant to the laser pulselength (Fig. 3). The density downramp in this region decreased the wake velocity by changing $\lambda_{p}$, putting the wake well above the trapping threshold without significant plasma modulation of the laser (which is an unstable process), and yielding stable trapping and acceleration to $\sim 1.5 \mathrm{MeV} / \mathrm{c}$. Absolute momentum spread was less than $200 \mathrm{keV} / \mathrm{c}$, as observed in experiments. The low trapping threshold also decreased transverse wake fields during trapping, producing a bunch with low transverse momen- 


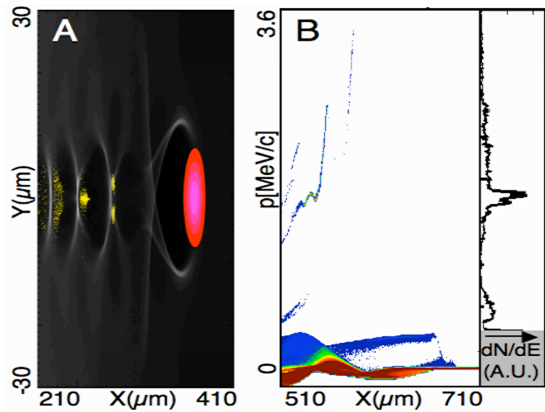

Figure 3: Particle simulations show that wave breaking on a plasma density downramp produces $\mathrm{MeV}$ electron bunches. At the location where the pulselength is near resonant with the density (A), the laser pulse (red) excites a large wake (density, grayscale). Variation of $\lambda_{p}$ (visible in the density) due to the ramp decreases wake velocity and trapping threshold, leading to trapping of an electron bunch (particles with $\mathrm{E}>0.5 \mathrm{MeV} / \mathrm{c}$, yellow). The bunch phase space $(\mathrm{B}$, red=maximum) shows a narrow momentum spread bunch at $1.5 \mathrm{MeV} / \mathrm{c}$. The lineout at the right side of the phase space shows the momentum spectrum.

tum of $\sim 50 \mathrm{keV} / \mathrm{c}$ (divergence $20 \mathrm{mrad}$, similar to experiment) compared with $300-3000 \mathrm{keV} / \mathrm{c}$ for homogeneous plasmas, and hence low emittance. Also reasonably consistent with experimental measurements, bunch charge is of order $0.2 \mathrm{nC}$, and $80-90 \%$ of the laser energy is transmitted while the mode is undistorted. Bunch size at formation is approximately $10 \mu \mathrm{m}$ long by $5 \mu \mathrm{m}$ diameter. Together with the experimental and simulated divergences, this indicates a normalized emittance of order $0.1-0.2 \pi$ $\mathrm{mm}-\mathrm{mrad}-\mathrm{MeV} / \mathrm{c}$. As $\lambda_{p}$ lengthens in the downramp, the bunch can expand to $\geq 50 \mu \mathrm{m}$ in dimension while within the plasma's space charge neutralization, explaining the absence of space-charge induced blow-up [21] of the experimental bunches and consistent with the experimental bunch length as meausured by $\mathrm{THz}$ diagnostics [22, 23]. Remaining differences with experiments are likely the result of the 2D nature of the simulations and parameter uncertainties.

Due to high transmission of the laser mode, if the downramp ends in a low density uniform plasma just after trapping, a wake can be excited by the transmitted laser to post accelerate the bunch to high energy. Because the bunch is $\sim 10 \mu \mathrm{m}$ in size at this point, initial simulations show it is efficiently trapped and accelerated. Previous simulations predict that post acceleration of electron bunches in plasma channels can nearly preserve momentum spread and emittance [24]. Such staging may enable greatly reduced relative momentum spread and emittance, potentially with 100 $\mathrm{keV} / \mathrm{c}$ class momentum spread and low transverse emittance at $\mathrm{GeV}$ and greater energies.

\section{CONCLUSION}

In conclusion, experiments demonstrated production of stable femtosecond electron bunches with $170 \mathrm{keV} / \mathrm{c}$ mo- mentum spread, 10 to 100 fold lower momentum spread than previous laser accelerators. The bunches have central momentum of $0.76 \mathrm{MeV} / \mathrm{c}, 20 \mathrm{keV} / \mathrm{c}(3 \%)$ momentum stability, and $2 \mathrm{mrad}$ pointing stability, and are order 30$100 \mathrm{fs}$ in duration. Charge stability of $15 \%$ RMS was observed. Such electron bunches may directly benefit applications such as ultrafast electron diffraction for chemistry. Experiments and simulations indicate that bunch emittance is of order $0.1 \pi \mathrm{mm}-\mathrm{mrad}-\mathrm{MeV} / \mathrm{c}$ and that the laser mode is well transmitted through the jet $(\sim 70 \%)$, and simulations indicate plasma down ramps can be coupled directly to a subsequent plasma for post acceleration. Such controlled injection is projected to produce stable bunches at $\mathrm{GeV}$ energies and beyond with $<0.1 \%$ energy spread, and to increase bunch energy by eliminating the need to operate the accelerating channel at high density to obtain injection. Such stable reduced momentum spread and emittance bunches are important to eventual collider or 4th generation light source (FEL) applications of LWFAs.

We appreciate contributions from D. Bruhwiler, J. van Tilborg, V. Leurent, S.A. Gaillard, P. Bach, M. Dickinson, D. Syversrud, J. Wallig, and N. Ybarrolaza.

\section{REFERENCES}

[1] T. Tajima and J. M. Dawson, Phys. Rev. Lett. 43, 267 (1979)

[2] E. Esarey et al., IEEE Trans. Plasma Sci. 24, 252 (1996).

[3] W. P. Leemans et al., Phys. Rev. Lett. 89, 4802 (2002).

[4] C. G. R. Geddes et al., Nature 431, 538 (2004).

[5] S. P. D. Mangles et al., Nature 431, 535 (2004).

[6] J. Faure et al., Nature 431, 541 (2004).

[7] W. P. Leemans et al., Nature Physics 2, 696 (2006).

[8] E. Esarey et al., Phys. Rev. Lett. 79, 2682 (1997).

[9] C. B. Schroeder et al., Phys. Rev. E 59, 6037 (1999).

[10] G. Fubiani et al., Phys. Rev. E 73, 026402 (2006).

[11] J. Faure et al., Nature 444, 737 (2006).

[12] R. G. Hemker, N. M. Hafz, and M. Uesaka, Phys. Rev. ST Accel. Beams 5, 041301 (2002).

[13] H. Suk et al., Phys. Rev. Lett. 86, 1011 (2001).

[14] S. Bulanov et al., Phys. Rev. E 58, R5257 (1998).

[15] C. G. R. Geddes et al., submitted to Phys. Rev. Lett. .

[16] LOASIS facility: http://loasis.lbl.gov/.

[17] C. G. R. Geddes et al., Phys. Rev. Lett. 95, 145002 (2005).

[18] W. P. Leemans et al., Phys. Plasmas 8, 2510 (2001).

[19] C. G. R. Geddes et al., Phys. Plasmas 12, 056709 (2005).

[20] C. Nieter and J. Cary, J. Comp. Phys. 196, 448 (2004).

[21] G. Fubiani et al., Phys. Rev. ST Accel. Beams 9, 064402 (2006).

[22] W. P. Leemans et al., Phys. Rev. Lett. 91, 074802 (2003).

[23] G.R. Plateau et al ,these proceedings.

[24] B. A. Shadwick, G. M. Tarkenton, and C. B. Schroeder, in Bull. Am. Phys. Soc. Plasma Physics Meeting 47 (Am. Phys. Soc., College Park MD, 2005), p. 213. 